\title{
Properties of the series solution for Painlevé I
}

A.N.W. Hone, O. Ragnisco, F. Zullo

To cite this article: A.N.W. Hone, O. Ragnisco, F. Zullo (2013) Properties of the series solution for Painlevé I, Journal of Nonlinear Mathematical Physics 20: Supplement 1, 85100, DOI: https://doi.org/10.1080/14029251.2013.862436

To link to this article: https://doi.org/10.1080/14029251.2013.862436

Published online: 04 January 2021 


\title{
Properties of the series solution for Painlevé I
}

\author{
A.N.W. Hone \\ School of Mathematics, Statistics \& Actuarial Science, University of Kent \\ Canterbury, Kent, U.K. \\ A.N.W.Hone@kent.ac.uk \\ O. Ragnisco \\ and \\ F. Zullo \\ Dipartimento di Fisica, Università Roma Tre, Via della Vasca Navale 84 \\ Roma, Italy. \\ ragnisco@fis.uniroma3.it \\ zullo@fis.uniroma3.it \\ Received 2 October 2012 \\ Accepted 20 June 2013
}

\begin{abstract}
We present some observations on the asymptotic behaviour of the coefficients in the Laurent series expansion of solutions of the first Painlevé equation. For the general solution, explicit recursive formulae for the Taylor expansion of the tau-function around a zero are given, which are natural extensions of analogous formulae for the elliptic sigma function, as given by Weierstrass. Numerical and exact results on the symmetric solution which is singular at the origin are also presented.
\end{abstract}

Keywords: Painlevé equation; tau-function; sigma function.

2000 Mathematics Subject Classification: 33E05, 33E17, 34M55

\section{Introduction}

The first Painlevé equation $\left(P_{I}\right)$ is usually written in the canonical form

$$
\frac{d^{2} u}{d z^{2}}=6 u^{2}+z
$$

It is known that all solutions of (1.1) are non-classical transcendental functions, in the sense that they cannot be written in terms of algebraic or elliptic functions of $z$, nor in terms of classical special functions defined by linear differential equations. The $P_{I}$ equation was discovered in Painlevé's classification of second order differential equations whose solutions have the property that all movable singularities are poles. For the equation (1.1), it is further known that all solutions of this equation are meromorphic, with an infinite number of poles in the complex plane. Although Painlevé's original approach to proving meromorphicity, as outlined in chapter XIV of Ince's book [10], appears to have some gaps from the modern point of view, these issues have subsequently been resolved (see [7]), and various other methods of proof are now available [5, 11, 19].

The papers in this special issue are written in honour of Okamoto's insights concerning the space of initial values for the Painlevé equations. The important point made by Okamoto is that, in 
order to consider the solutions of a system with poles, such as 1.1 , one should enlarge the phase space: as well as regular initial conditions $\left(u\left(z_{0}\right), u^{\prime}\left(z_{0}\right)\right) \in \mathbb{C}^{2}$, it is necessary to consider the case where $u$ (hence also $u^{\prime}$ ) has a pole at $z=z_{0}$; this requires adding points at infinity to the phase space, and leads to a sequence of blowups around singular points [2].

Boutroux showed that, for large $|z|$, with the scaled variables $U=z^{-1 / 2} u$ and $Z=\frac{4}{5} z^{5 / 4}$, the solution of (1.1) behaves asymptotically like an elliptic function, $U \sim \wp$, where $\wp$ is the Weierstrass function, which satisfies the second order differential equation

$$
\wp^{\prime \prime}=6 \wp \wp^{2}-\frac{1}{2} g_{2}
$$

The purpose of this article is to present some remarks about the direct comparison between the solutions of the $P_{I}$ equation and the Weierstrass elliptic functions, at the level of exact series expansions rather than asymptotics.

After introducing our conventions of scaling and notation in the next section, in section 3 we proceed to compare the coefficients in the Laurent series for the $\wp$ function, which are written in terms of modular forms, with the corresponding expansion of solutions of 1.1 around a pole. This produces a natural analogy between the lemniscatic and equianharmonic elliptic functions, which have the symmetries of the square and the hexagon, respectively, and a special solution of $P_{I}$ which is singular at the origin and has pentagonal symmetry. In section 4 we apply the same idea to the tau-function of $P_{I}$, and show how both the Hirota bilinear form, and an associated equation of degree four, lead to recursive formulae for the Taylor coefficients in the expansion around a zero. This both generalizes the results of Eilbeck and Enolskii on the Weierstrass sigma function [3], and at the same time extends some classical expansion formulae due to Weierstrass [22] to the case of $P_{I}$. As well as being of theoretical interest, we show that these formulae are also useful for doing numerical computations.

\section{The equation with parameters}

The programme of classification initiated by Painlevé began by considering the necessary conditions for an equation such as (1.1) to have only poles as movable singularities. This requires some local analysis to identify the form that algebraic singularities can take at leading order, followed by a power series expansion around any such singularity to check whether any secular terms can appear; nowadays this procedure is often referred to as Painlevé analysis (see [8] and references). For the equation (1.1) the only algebraic singularities are double poles, $y \sim\left(z-z_{0}\right)^{-2}$, where $z_{0}$ is the (movable) position of the pole. This leading order behaviour then extends to a power series, whose resonances (the places where arbitrary coefficients can appear in the expansion) are found by substituting $u \sim\left(z-z_{0}\right)^{-2}\left(1+\varepsilon\left(z-z_{0}\right)^{r}\right)$ into the equation and comparing the leading order terms that are linear in $\varepsilon$. In this case one finds a quadratic equation in $r$, with roots $-1,6$; the root $r=-1$ corresponds to the fact that the position of the pole at $z_{0}$ is movable, while the value $r=6$ means that the coefficient of $\left(z-z_{0}\right)^{4}$ in the expansion should be arbitrary. Finally, one must calculate the terms of a full Laurent expansion around the pole up to order $\left(z-z_{0}\right)^{4}$, and check that no additional counterterms involving $\log \left(z-z_{0}\right)$ are needed for consistency. Hence one obtains a local series representation of the general solution, with two free constants corresponding to the resonances. Assuming that such a Laurent expansion is convergent in some neighbourhood of $z_{0}$,

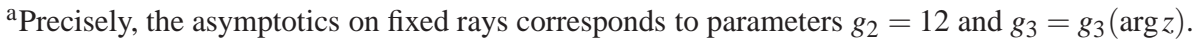


this verifies that 1.1 has solutions that are locally meromorphic around a pole. Then for $P_{I}$, any local Laurent expansion around a pole completely fixes a global solution of the equation, by analytic continuation. However, purely local analysis is not sufficient to establish that all solutions are meromorphic in $\mathbb{C}$, since global estimates are necessary to ensure that poles cannot coalesce to form an essential singularity (see e.g. [19]).

For what follows, it is worth mentioning at this stage that the details of the Painlevé analysis for the differential equation (1.2) are almost identical to those for $P_{I}$. However, if one replaces the $z$ in (1.1), or the constant $-\frac{1}{2} g_{2}$ in (1.2), by an arbitrary holomorphic function $f(z)$, then at the resonance $r=6$ one requires $f^{\prime \prime}\left(z_{0}\right)=0$ for all $z_{0}$, and hence $f$ must be a linear function, in order to have a consistent Laurent series.

In the rest of the paper we consider solutions of (1.1) with a pole at $z_{0}$, so it is convenient to replace $z \rightarrow z+z_{0}$, which adds a constant to the right hand side of (1.1). Furthermore, for comparison with the equation (1.2) it is useful to rescale $u \rightarrow(-6 \lambda)^{-2 / 5} u$ and $z \rightarrow(-6 \lambda)^{1 / 5} z$, so that we arrive at the following equation:

$$
\frac{d^{2} u}{d z^{2}}=6 u^{2}-6 \lambda z-\frac{g_{2}}{2}
$$

The above equation has two parameters, namely $\lambda$ and $g_{2}$, and any solution of the $P_{I}$ equation in the canonical form (1.1) with a double pole at $z=z_{0}$ corresponds to a solution of (2.1) with a pole at $z=0$, for a suitable choice of the constant $g_{2}$, with any $\lambda \neq 0$. Moreover, when $\lambda=0$ the equation (2.1) reduces to (1.2).

\subsection{Recursion for Laurent coefficients}

To determine the local Laurent expansion of the solution of (2.1) with a double pole at the origin, we insert the expression

$$
u(z)=\sum_{n=0} c_{n} z^{n-2}, \quad c_{0}=1
$$

into the equation, from which subsequent coefficients are determined by the recursion

$$
(n+1)(n-6) c_{n}=6 \sum_{j=1}^{n-1} c_{j} c_{n-j}-\frac{1}{2} g_{2} \delta_{n, 4}-6 \lambda \delta_{n, 5}, \quad n \geq 1
$$

The above relation determines all the coefficients $c_{1}, \ldots, c_{5}$ uniquely, but $c_{6}$ is arbitrary (corresponding to the aforementioned resonance). Once $c_{6}$ has been fixed, then all $c_{n}$ for $n \geq 7$ are uniquely determined by (2.3). We find

$$
c_{1}=c_{2}=c_{3}=0, \quad c_{4}=\frac{g_{2}}{20}, \quad c_{5}=\lambda, \quad c_{6}=\frac{g_{3}}{28}, \quad c_{7}=0, \quad \ldots,
$$

where the value of $c_{6}$ is given in terms of an additional parameter $g_{3}$, which is introduced for ease of comparison with the elliptic case. The following result is immediate.

Lemma 2.1. For $n \geq 1$ the coefficients of the expansion (2.2), with $c_{6}=g_{3} / 28$, are polynomials in $g_{2}, \lambda$ and $g_{3}$, i.e. $c_{n}=\mathrm{P}_{n}\left(g_{2}, \lambda, g_{3}\right)$, where $\mathrm{P}_{n}$ has rational coefficients and is weighted homogeneous 
of total degree $n$ in these arguments with weights 4,5 and 6 respectively:

$$
\mathrm{P}_{n}\left(\zeta^{4} g_{2}, \zeta^{5} \lambda, \zeta^{6} g_{3}\right)=\zeta^{n} \mathrm{P}_{n}\left(g_{2}, \lambda, g_{3}\right) \quad \forall \zeta \in \mathbb{C}^{*} .
$$

The polynomials $\mathrm{P}_{n}$ for $n=1, \ldots, 7$ can be read off from (2.4). Note that, a priori, they must be identically zero for $n=1,2,3,7$, since there are no non-zero homogeneous polynomials with these weights; but they are non-zero for all other values of $n$. Here we list the next few non-trivial ones:

$$
\begin{gathered}
\mathrm{P}_{8}=\frac{g_{2}^{2}}{1200}, \quad \mathrm{P}_{9}=\frac{g_{2} \lambda}{50}, \quad \mathrm{P}_{10}=\frac{3}{22}\left(\lambda^{2}+\frac{g_{2} g_{3}}{280}\right), \quad \mathrm{P}_{11}=\frac{g_{3} \lambda}{140}, \\
\mathrm{P}_{12}=\frac{1}{208}\left(\frac{g_{2}^{3}}{750}+\frac{g_{3}^{2}}{49}\right), \quad \mathrm{P}_{13}=\frac{11 g_{2}^{2} \lambda}{49000}, \quad \mathrm{P}_{14}=\frac{1}{44}\left(\frac{59 g_{2} \lambda^{2}}{500}+\frac{g_{3} g_{2}^{2}}{4200}\right) .
\end{gathered}
$$

These polynomials have some interesting properties. As will be explained in section 3 , for $\lambda=0$ the polynomials $\mathrm{P}_{2 n}\left(g_{2}, 0, g_{3}\right)$ are polynomials in $g_{2}$ and $g_{3}$, which define modular forms: up to overall scaling they give the Eisenstein series of the elliptic curve

$$
y^{2}=4 x^{3}-g_{2} x-g_{3} .
$$

In fact, viewing each $\mathrm{P}_{n}$ as a polynomial in $\lambda$ with coefficients in $\mathbb{Q}\left[g_{2}, g_{3}\right]$, each coefficient is weighted homogeneous in $g_{2}, g_{3}$ with some weight, so also defines a modular form. Thus we refer to the $\mathrm{P}_{n}$ as modular polynomials.

Henceforth we let $u\left(z ; g_{2}, \lambda, g_{3}\right)$ denote the meromorphic function defined by the series (2.2). Our main task in section 3 will be to consider the asymptotic behaviour of the coefficients $c_{n}$ in this series as $n \rightarrow \infty$, and the way that this depends on the parameters $g_{2}, \lambda, g_{3}$. Before doing this, we introduce some objects needed in section 4 .

\subsection{Hamiltonian and tau-function}

Hamiltonian forms of the Painlevé equations were originally found by Malmquist [15]. Some years later, Okamoto introduced the notion of a tau-function associated with the Hamiltonian [16].

The equation (2.1) can be written as a Hamiltonian system like so:

$$
u^{\prime}=\frac{\partial h}{\partial v}, \quad v^{\prime}=-\frac{\partial h}{\partial u}, \quad \text { with } \quad h=\frac{1}{2} v^{2}-2 u^{3}+\frac{1}{2} g_{2} u+6 \lambda z u+\frac{1}{2} g_{3} .
$$

(The constant $g_{3}$ is included in the Hamiltonian $h$ for later convenience.) Taking the total derivative of $h$ with respect to $z$ we have

$$
h^{\prime}=\frac{\partial h}{\partial z}=6 \lambda u .
$$

The tau-function associated with a solution of (2.1) is a function $\tau=\tau(z)$ such that

$$
u=-\frac{d^{2}}{d z^{2}} \log \tau .
$$

Observe that the preceding relation only defines $\tau$ up to gauge transformations

$$
\tau \rightarrow \exp (a z+b) \tau, \quad a, b \quad \text { arbitrary, }
$$

and the fact that $u$ is meromorphic means that $\tau$ is holomorphic, with $\tau$ having simple zeros wherever $u$ has double poles. 
We also note from (2.7) that, up to the addition of a constant, we have

$$
h=-6 \lambda \frac{d}{d z} \log \tau .
$$

The constant of integration has been fixed so that when $\lambda=0$ we have $h=0$, and the right hand side of (2.6) corresponds to the first order differential equation for the Weierstrass $\wp$ function, i.e.

$$
\left(\wp^{\prime}\right)^{2}=4 \wp^{3}-g_{2} \wp-g_{3},
$$

for $u=\wp$ and $v=\wp{ }^{\prime}$. This choice of integration constant will also be convenient for the analysis of the tau-function when $\lambda \neq 0$.

\section{Asymptotics of coefficients in the Laurent series}

We now describe the behaviour of the coefficients $c_{n}$, which satisfy the recursion

$$
c_{n}=\frac{6}{(n+1)(n-6)} \sum_{k=1}^{n-1} c_{k} c_{n-k}, \quad n \geq 7,
$$

for a suitable choice of initial values. To begin with we consider the classical case $\lambda=0$, with $u\left(z ; g_{2}, 0, g_{3}\right)=\wp\left(z ; g_{2}, g_{3}\right)$, for which $c_{1}=c_{2}=c_{3}=c_{5}=0$ in (3.1).

\subsection{The elliptic case}

The Weierstrass $\wp$ function can be defined as the unique solution of the first order differential equation (2.11) having a double pole at $z=0$. However, in most standard treatments of elliptic function theory (see e.g. chapter XX in [23]), it is usually defined by its Mittag-Leffler expansion, that is

$$
\wp\left(z ; g_{2}, g_{3}\right)=\frac{1}{z^{2}}+\sum_{\Omega \in \Lambda \backslash\{0\}}\left(\frac{1}{(z-\Omega)^{2}}-\frac{1}{\Omega^{2}}\right),
$$

where $\Lambda$ denotes the period lattice, consisting of all periods of the form $\Omega=2 m \omega_{1}+2 n \omega_{2}$ for $(m, n) \in \mathbb{Z}^{2}$, generated by the two independent periods $2 \omega_{1}, 2 \omega_{2}$. The uniform convergence of the expansion (3.2) is guaranteed by the fact that the power sums $\sum_{\Omega \neq 0} \Omega^{-\mu}$ are absolutely convergent for all $\mu>2$, and expanding around $z=0$ gives the series (2.2) with $c_{0}=1, c_{1}=c_{2}=0$ and

$$
c_{n}=(n-1) \sum_{\Omega \neq 0} \Omega^{-n}, \quad n \geq 3 .
$$

Thus it follows from (3.2) that $\wp$ satisfies the differential equation (2.11) with

$$
g_{2}=60 \sum_{\Omega \neq 0} \Omega^{-4}, \quad g_{3}=140 \sum_{\Omega \neq 0} \Omega^{-6}
$$

Conversely, given a solution of (2.11) with parameters $g_{2}, g_{3}$, two independent periods are obtained from the integrals $2 \omega_{j}=\oint_{\mathfrak{c}_{j}} \frac{d x}{y}, j=1,2$ around cycles $\mathfrak{c}_{1}, \mathfrak{c}_{2}$ that generate the homology of the elliptic curve (2.5). (This is in the generic case $g_{2}^{3}-27 g_{3}^{2} \neq 0$; otherwise (2.11) is solved in elementary functions.) 
Observe that, from the symmetry $\Lambda=-\Lambda$ of the lattice where the poles of $\wp(z)$ are situated, the odd index coefficients $c_{2 n+1}=0$ for all $n \geq 1$ (hence $\wp$ is an even function). The even index sums (3.3) can be rewritten as

$$
c_{2 n}=\frac{2 n-1}{\left(2 \omega_{1}\right)^{2 n}} G_{2 n}(\tau)
$$

where the function $G_{2 n}(\tau)$ is the Eisenstein series, a modular form of weight $2 n$ corresponding to a normalized lattice with periods $1, \tau$, with $\tau=\frac{\omega_{2}}{\omega_{1}}$ :

$$
G_{2 n}(\tau)=\sum_{(p, q) \in \mathbb{Z}^{2} \backslash\{(0,0)\}} \frac{1}{(p+\tau q)^{2 n}} .
$$

The periods $2 \omega_{1}, 2 \omega_{2}$ can always be ordered so that $\operatorname{Im} \tau>0$, and by choosing $\left|2 \omega_{1}\right|$ to be minimal we also have $|\tau| \geq 1$. Then the series (2.2) has radius of convergence $\left|2 \omega_{1}\right|$, and the asymptotic behaviour of $c_{2 n}$ depends on $\tau$. There are two possibilities:

- $|\tau|>1: \lim _{n \rightarrow \infty} G_{2 n}(\tau)=2$;

- $|\tau|=1: G_{2 n}(\tau)$ has no limit as $n \rightarrow \infty$.

Note that we can always choose $\tau$ so that it lies in the fundamental domain $\mathscr{F}=\{|\tau| \geq 1\} \cap$ $\left\{|\operatorname{Re} \tau| \leq \frac{1}{2}\right\}$ ( [18], Proposition 1.5). In the first situation, \pm 1 are the only normalized periods that lie on the unit circle, so all of the terms in the sum (3.5) vanish as $n \rightarrow \infty$ apart from the contribution from $(p, q)=( \pm 1,0)$. For the second possibility, $\tau=e^{\mathrm{i} \theta}$ lies on the lower boundary of $\mathscr{F}$, and (by using the modular transformation $\tau \rightarrow-1 / \tau)$ it can always be assumed that $\pi / 3 \leq \theta \leq \pi / 2$; then $\theta=\pi / 3$, with six normalized periods on the unit circle (the sixth roots of unity), or otherwise there are four periods $\pm 1, \pm \tau$ with modulus one. The extreme cases are the so called equianharmonic case $(\theta=\pi / 3)$, and the lemniscatic case $(\theta=\pi / 2)$.

Equianharmonic case: This corresponds to $g_{2}=0$ in 2.11). Without loss of generality (by rescaling) one may set $g_{3}=1$ and find half-periods

$$
\omega_{1}=\int_{\sqrt[3]{4}}^{\infty} \frac{d x}{\sqrt{4 x^{3}-1}}=\frac{\Gamma^{3}\left(\frac{1}{3}\right)}{4 \pi}, \quad \omega_{2}=e^{\frac{i \pi}{3}} \omega_{1}
$$

which gives $\tau=e^{\frac{\mathrm{i} \pi}{3}}$. Then from the recurrence (3.1) with $c_{4}=0$ it follows that $G_{2 n}\left(e^{\frac{\mathrm{i} \pi}{3}}\right)=0$ unless $n=0 \bmod 3$. The Laurent series for the $\wp$ function becomes

$$
\wp(z ; 0,1)=\frac{1}{z^{2}}+\sum_{n=1}^{\infty} \frac{(6 n-1)}{\left(2 \omega_{1}\right)^{6 n}} G_{6 n}\left(e^{\frac{\mathrm{i} \pi}{3}}\right) z^{6 n-2}
$$

with $\omega_{1}$ as in (3.6). There are six normalized periods $e^{\frac{\mathrm{i} j \pi}{3}}, j=0, \ldots, 5$ on the unit circle, so in the limit of the Eisenstein series (3.5), only six terms survive to yield $\lim _{n \rightarrow \infty} G_{6 n}\left(e^{\frac{\mathrm{i} \pi}{3}}\right)=6$. Some values of these modular functions are given to twenty decimal places in Table 1 . 


\begin{tabular}{|c|c|}
\hline$n$ & $G_{6 n}\left(e^{\frac{1}{3}}\right)$ \\
\hline \hline 1 & 5.86303169342540159797 \\
2 & 6.00963997169768048102 \\
3 & 5.99971835637052593409 \\
4 & 6.00001164757977973485 \\
5 & 5.99999958743553301523 \\
6 & 6.00000001557436652006 \\
$\ldots$ & $\ldots$ \\
11 & 5.99999999999999892076 \\
12 & 6.00000000000000003997 \\
13 & 5.99999999999999999851 \\
14 & 6.00000000000000000005 \\
\hline
\end{tabular}

Table 1: The oscillating values of $G_{6 n}\left(e^{\frac{\mathrm{i} \pi}{3}}\right)$.

Lemniscatic case: This case corresponds to $g_{3}=0$, and (upon scaling so that $g_{2}=4$ ) the halfperiods are found as

$$
\omega_{1}=\int_{0}^{1} \frac{d t}{\sqrt{1-t^{4}}}=\frac{1}{4} B(1 / 4,1 / 2), \quad \omega_{2}=\mathrm{i} \omega_{1},
$$

whence $\tau=\mathrm{i}$. The fact that $c_{6}=0$ in (3.1) now implies that $G_{2 n}(\mathrm{i})=0$ unless $n$ is even, so that the series (2.2) in this case takes the form

$$
\wp(z ; 4,0)=\frac{1}{z^{2}}+\sum_{n=1}^{\infty} \frac{4 n-1}{\left(2 \omega_{1}\right)^{4 n}} G_{4 n}(\mathrm{i}) z^{4 n-2} .
$$

The coefficients can also be expressed in terms of the Hurwitz numbers $H_{n}$ [1, 9, 13] (elliptic analogues of the Bernoulli numbers) which are given by $H_{n}=\frac{(4 n) !}{\left(4 \omega_{1}\right)^{4 n}} G_{4 n}(\mathrm{i})$. From 3.1 it follows that these numbers satisfy the recurrence found by Hurwitz:

$$
H_{n}=\frac{3}{(2 n-3)\left(16 n^{2}-1\right)} \sum_{k=1}^{n-1}(4 k-1)(4 n-4 k-1)\left(\begin{array}{c}
4 n \\
4 k
\end{array}\right) H_{k} H_{n-k}
$$

As is suggested by Table 2, the limit of the Eisenstein series (3.5) in this case is $\lim _{n \rightarrow \infty} G_{4 n}(\mathrm{i})=4$.

\subsection{The general case $\lambda \neq 0$}

All solutions of $P_{I}$ are known to have order of growth 5/2 [20], and this is the same as the infimum of the values of $\mu$ such that the power sums over non-zero poles, $\sum_{\Omega \neq 0} \Omega^{-\mu}$, are absolutely convergent ( [21], chapter VIII). As a consequence, the solutions of [2.1] with $\lambda \neq 0$ admit a Mittag-Leffler expansion of the same form (3.2) as for $\wp(z)$ :

$$
u\left(z ; g_{2}, \lambda, g_{3}\right)=\frac{1}{z^{2}}+\sum_{\substack{\text { poles } \Omega \\ \Omega \neq 0}}\left(\frac{1}{(z-\Omega)^{2}}-\frac{1}{\Omega^{2}}\right)
$$




\begin{tabular}{|c|c|}
\hline$n$ & $G_{4 n}(\mathrm{i})$ \\
\hline \hline 1 & 3.15121200215389753821 \\
2 & 4.25577303536518951844 \\
3 & 3.93884901282797037475 \\
4 & 4.01569503302502485587 \\
5 & 3.99609675317628955957 \\
6 & 4.00097680530383862810 \\
$\ldots$ & $\ldots$ \\
11 & 3.99999904632591103400 \\
12 & 4.00000023841859318284 \\
13 & 3.99999994039535611558 \\
14 & 4.00000001490116124950 \\
\hline
\end{tabular}

Table 2: The oscillating values of $G_{4 n}(\mathrm{i})$.

(cf. [20], Theorem 7.1). The main difference with the elliptic case is that the poles of $u$ no longer lie on a lattice, and suitable analogues of elliptic integrals to determine the positions of the poles are unavailable.

Nevertheless, the formula (3.3) for the coefficients in (2.2) still holds in general, and as a consequence, from (2.3), $c_{3}=0=c_{7}$ gives $\sum_{\Omega \neq 0} \Omega^{-3}=0=\sum_{\Omega \neq 0} \Omega^{-7}$; while generically one expects that $c_{n} \neq 0$ for $n \geq 4, n \neq 7$ (and similarly for the corresponding power sums). Following the elliptic case, by picking a non-zero pole $\Omega_{*}$ such that $\left|\Omega_{*}\right|$ is minimal, one may write

$$
c_{n}=\frac{n-1}{\Omega_{*}^{n}} F_{n}, \quad F_{n}=\sum_{\hat{\Omega} \neq 0} \hat{\Omega}^{-n},
$$

where the sum is over the non-zero poles $\hat{\Omega}=\Omega / \Omega_{*}$ of the rescaled solution $u\left(z ; \hat{g}_{2}, \hat{\lambda}, \hat{g}_{3}\right)$ with $\hat{g}_{2}=\Omega_{*}^{4} g_{2}, \hat{\lambda}=\Omega_{*}^{5} \lambda, \hat{g}_{3}=\Omega_{*}^{6} g_{3}$. Generically, for the rescaled solution, there should be only one pole with modulus 1 (corresponding to $\Omega_{*}$ ), with all other non-zero poles lying outside the unit circle. Assuming this to be the case, it follows that $\lim _{n \rightarrow \infty} F_{n}=1$, whence $\Omega_{*}=\lim _{n \rightarrow \infty} \frac{c_{n}}{c_{n+1}}$. Thus, in the generic situation, numerical iteration of (3.1) allows the pole nearest to $z=0$ to be calculated very efficiently.

The question of precisely when a non-generic solution can arise, with two or more poles $\Omega$ having the same modulus $\left|\Omega_{*}\right|$, seems to be a very difficult one. However, there is one situation where we know this to be so, namely when $g_{2}=0=g_{3}$ : we call this the pentagonal case. It corresponds to a solution which is invariant under the order 5 scaling symmetry of $P_{I}$, which is generated by taking $z \rightarrow e^{2 \pi \mathrm{i} / 5} z, u \rightarrow e^{6 \pi \mathrm{i} / 5} u$ in equation (1.1). There are precisely two such solutions, and it appears that so far these are the only solutions of $P_{I}$ for which the monodromy data can be explicitly related to the initial conditions at $z=0$ [12]: one has a double pole, and the other has a triple zero at the origin; it is the former symmetric solution which is of interest to us here.

Pentagonal case: When $g_{2}$ and $g_{3}$ both vanish, it follows from (2.3) that $c_{n}=0$ unless $n=0 \bmod 5$, so that the Laurent series (2.2) for $\tilde{u}(z)=u(z ; 0, \lambda, 0)$ takes the form

$$
\tilde{u}(z)=\frac{1}{z^{2}}+\sum_{n=1} \frac{5 n-1}{\gamma^{n}} \tilde{F}_{n} \lambda^{n} z^{5 n-2},
$$


where we set $v_{n}=c_{5 n}=\frac{(5 n-1)}{\gamma^{n}} \tilde{F}_{n}$. The coefficients $v_{n}$ satisfy the recurrence

$$
v_{n}=\frac{6}{(5 n+1)(5 n-6)} \sum_{k=1}^{n-1} v_{k} v_{n-k}, \quad n \geq 2, \quad \text { with } \quad v_{1}=1 .
$$

In this case, the non-zero poles of the solution (3.12) lie on regular pentagons centred at $z=0$ : when $\Omega$ is a pole of $\tilde{u}$, then so is $e^{\frac{2 \pi i j}{5}} \Omega$ for $j=1,2,3,4$. We have $F_{n}=0$ if $n \neq 0$ mod 5, and from (3.11), with 5 poles on the unit circle, $\lim _{n \rightarrow \infty} F_{5 n}=5$. At $n=30$ we find a value of $\gamma$ apparently correct to 23 decimal places:

$$
\gamma=\lim _{n \rightarrow \infty} \frac{(5 n+4) v_{n}}{(5 n-1) v_{n+1}} \approx 18.32138268472483887119960 \ldots
$$

Given this accuracy in $\gamma$, the quantities $\tilde{F}_{n}=F_{5 n}$ can then be calculated, and should converge exponentially fast to the value 5 ; see Table 3 . The value $\gamma^{1 / 5} \approx 1.788923$ gives the non-zero real pole of the solution $u(z ; 0,1,0)$ closest to the origin, and the radius of convergence of the series (3.12); for an independent verification of this see section 4 .

The preceding results on limiting values of the coefficients in the symmetric cases can be summarized as follows.

Proposition 3.1. The solutions (3.10) for which only one of the parameters $g_{2}, \lambda, g_{3}$ is non-zero are invariant under the scaling $z \rightarrow \xi_{z}, u \rightarrow \xi^{-2} u$, where $\xi$ is a kth root of unity for $k=4,5,6$ respectively, and the normalized coefficients (3.11) satisfy

$$
\lim _{n \rightarrow \infty} F_{k n}=k \text {. }
$$

Proof: The normalized series $F_{n}=\sum_{\hat{\Omega} \neq 0} \hat{\Omega}^{-n}$ are all absolutely convergent (uniformly in $n$ ), and for a solution invariant under the symmetry of order $k$, the sum of each series vanishes unless $k \mid n$. With the chosen normalization, there are $k$ poles at the roots of unity $\hat{\Omega}=\xi^{j}, j=0, \ldots, k-1$. Given that there are no other poles on the unit circle, we find $F_{k n}=k+\sum_{|\hat{\Omega}|>1} \hat{\Omega}^{-k n}$, and hence (upon selecting the term $\hat{\Omega}=\tilde{\Omega}$ with smallest modulus outside the unit circle) $\left|F_{k n}-k\right|=\left|\tilde{\Omega}^{-k n} \sum_{|\hat{\Omega}|>1}(\tilde{\Omega} / \hat{\Omega})^{k n}\right| \leq$ $|\tilde{\Omega}|^{-k n} \sum_{|\hat{\Omega}|>1}|\tilde{\Omega} / \hat{\Omega}|^{k} \rightarrow 0$ exponentially fast as $n \rightarrow \infty$.

The only statement above that requires further justification is the assertion that there are no other poles on the unit circle for the case $k=5$. (Note that for $k=4,6$ this is obvious from the properties of the pole lattice.) A numerical verification of this fact is given in the next section (see Figure 1). We conclude this section with some observations on the cases $g_{2}=0$ and $g_{3}=0$.

\subsubsection{The case $g_{2}=0$}

To consider the form of the quantities $c_{n}$ when $g_{2}=0$, it is convenient to use the parameter $\alpha=$ $g_{3} / 28$. Thus we have the recursion (3.1) with $c_{0}=1, c_{1}=c_{2}=c_{3}=c_{4}=0, c_{5}=\lambda$ and $c_{6}=\alpha$, and find that the structure of the iterates depends on the index $\bmod 5$, so that

$$
c_{5 n+p}=\sum_{m=0}^{\left[\frac{n-p}{6}\right]} c_{5 n+p}^{(m)} \alpha^{5 m+p} \lambda^{n-6 m-p}, \quad p=0, \ldots, 4,
$$

for certain rational numbers $c_{5 n+p}^{(m)}$, which satisfy quadratic recurrence relations. 


\begin{tabular}{|c|c|}
\hline$n$ & $\tilde{F}_{n}$ \\
\hline \hline 1 & 4.58034567118120971779 \\
2 & 5.08595550727477491732 \\
3 & 4.99187877676419618477 \\
4 & 5.00112762186482314743 \\
5 & 4.99986996982708054870 \\
6 & 5.00001616272241466829 \\
$\ldots$ & $\ldots$ \\
11 & 4.99999999957591996469 \\
12 & 5.00000000005151463070 \\
13 & 4.99999999999374379484 \\
14 & 5.00000000000075986460 \\
\hline
\end{tabular}

Table 3: The oscillating values of $\tilde{F}_{n}=F_{5 n}$.

To begin with, we examine $c_{5 n}^{(0)}$, which are the coefficients of $\alpha^{0}$. Upon setting $v_{n} \doteq c_{5 n}^{(0)}$, we see that $v_{n}$ satisfies (3.13). The next terms to consider are $c_{5 n+1}^{(0)}$, i.e. the coefficients of $\alpha^{1}$, and upon setting $w_{n} \doteq c_{5 n+1}^{(0)}$ we find

$$
w_{n}=\frac{12}{(5 n+2)(5 n-5)} \sum_{k=1}^{n-1} w_{k} v_{n-k}, \quad n \geq 2, \quad \text { with } \quad w_{1}=1
$$

Observe that, supposing the sequence of $v_{n}$ to be known, the above recurrence is linear in the unknowns (unlike the recurrence (3.13) for the $v_{n}$ themselves). This means that the generating function for $w_{n}$ satisfies a second order linear ODE, within which the generating function of the $v_{n}$ appears as a coefficient.

As we shall now see, this property extends to the recursive generation of all the sequences $c_{5 n+p}^{(m)}$ when $(m, p) \neq(0,0)$. The linear recurrence solved by the general term $c_{5 n+p}^{(m)}$ can be written explicitly as

$$
c_{5 n+p}^{(m)}=K_{n, p} \sum_{k=1}^{n-1}\left(\sum_{j=0}^{m} \sum_{\ell=0}^{p} c_{5 k+\ell}^{(j)} c_{5(n-k)+p-\ell}^{(m-j)}+\sum_{j=0}^{m-1} \sum_{\ell=1}^{4-p} c_{5 k+\ell+p}^{(j)} c_{5(n-k)-\ell}^{(m-j-1)}\right), \quad n \geq 2,
$$

where $K_{n, p}$ is given by

$$
K_{n, p}=\frac{6}{(5 n+p+1)(5 n+p-6)}, \quad p=0,1,2,3,4 .
$$

The initial conditions for the equations (3.17) are that $c_{k}^{(m)}=0$ for $1 \leq k \leq 9$ and for all $m \geq 0$, except for $c_{5}^{(0)}=c_{6}^{(0)}=1$. From (3.17) it appears that all the nonlinearity in the problem of determining these coefficients is moved into finding the solution of 3.13 .

For the recurrence (3.13), note that it is sufficient to determine a single non-vanishing solution, since all other solutions can be obtained by the rescaling $v_{n} \rightarrow A^{n} v_{n}$, with $A$ arbitrary. The $v_{n}$ correspond precisely to the coefficients in the Laurent expansion (3.12) of the solution $\tilde{u}(z)$ 
with pentagonal symmetry that was discussed previously. To see an example of how this is related to the other coefficients, we consider $w_{n}=c_{5 n+1}^{(0)}$ once more, and introduce the generating function $G(x)=\sum_{n=1}^{\infty} w_{n} x^{n-1}$. From (3.16) it follows that $G$ satisfies $x G^{\prime \prime}+\frac{12}{5} G^{\prime}=\frac{12}{25} G \psi$, where $\psi(x)=\sum_{n=1}^{\infty} v_{n} x^{n-1}$. In fact, up to some scaling and shifting, $\psi(x)$ is just given by the pentagonal solution $\tilde{u}$; to be precise, $x \psi(x)=(x / \lambda)^{\frac{2}{5}} \tilde{u}\left((x / \lambda)^{\frac{1}{5}}\right)-1$. In a similar way, via (3.17), the generating functions for the other terms $c_{5 n+p}^{(m)}$ are related to each other: for example, that of $c_{5 n+2}^{(0)}$ is related to the generating functions of the sequences $c_{5 n}^{(0)}$ and $c_{5 n+1}^{(0)}$; and that of $c_{5 n}^{(1)}$ is related to the generating functions of the sequences $c_{5 n+p}^{(0)}, p=0, \ldots, 4$, and so on.

\subsubsection{Case $g_{3}=0$}

For the case $g_{3}=0$, it is helpful to introduce the parameter $\beta=g_{2} / 20$, and take the solution of (3.1) with $c_{0}=1, c_{1}=c_{2}=c_{3}=c_{6}=0, c_{5}=\lambda$ and $c_{4}=\beta$. The coefficients of (2.2) now have the structure

$$
c_{5 n-p}=\sum_{m=0}^{\left[\frac{n-p}{4}\right]} \hat{c}_{5 n-p}^{(m)} \beta^{5 m+p} \lambda^{n-4 m-p}, \quad p=0, \ldots, 4
$$

for some rational numbers $\hat{c}_{5 n-p}^{(m)}$.

The quantities $\hat{c}_{5 n}^{(0)}$, which appear as coefficients of $\beta^{0}$, are precisely the same as the numbers $v_{n}=c_{5 n}^{(0)}$ found previously, since setting $\beta=0$ just gives the pentagonal solution $\tilde{u}$ with the expansion (3.12). The next terms to consider are $\hat{w}_{n} \doteq \hat{c}_{5 n-1}^{(0)}$, the coefficients of $\beta^{1}$, which satisfy the recurrence

$$
\hat{w}_{n}=\frac{12}{(5 n)(5 n-7)} \sum_{k=1}^{n-1} \hat{w}_{k} v_{n-k} \quad n \geq 2, \quad \text { with } \quad \hat{w}_{1}=1
$$

Just as for the case $g_{2}=0$, once $v_{n}$ is given, this recurrence is linear in the unknowns $\hat{w}_{k}$. Analogously to equation (3.17), it is possible to write down the general recurrence solved by the term $\hat{c}_{5 n-p}^{(m)}$, the result being

$$
\hat{c}_{5 n-p}^{(m)}=K_{n,-p}\left(\sum_{j=0}^{m} \sum_{\ell=0}^{p} \sum_{k=1}^{n-1} \hat{c}_{5 k-\ell}^{(j)} \hat{c}_{5(n-k)+\ell-p}^{(m-j)}+\sum_{j=0}^{m-1} \sum_{\ell=1}^{4-p} \sum_{k=1}^{n} \hat{c}_{5 k-\ell-p}^{(j)} \hat{c}_{5(n-k)+\ell}^{(m-j)}\right),
$$

where again $K_{n, p}$ is given by (3.18), but above it appears with $p \rightarrow-p$ compared with (3.17). The formula (3.21) holds for $n \geq 2$, and the initial conditions are given by $\hat{c}_{k}^{(m)}=0$ for $1 \leq k \leq 6$ and for all $m \geq 0$, except for $\hat{c}_{4}^{(0)}=\hat{c}_{5}^{(0)}=1$. Similarly to the situation for $g_{2}=0$, linear ODEs for the generating functions of the rational numbers $\hat{c}_{5 n-p}^{(m)}$ for $(m, p) \neq(0,0)$ can be constructed recursively, once the numbers $v_{n}$ are known.

\section{Expansion of the tau-function}

The tau-function for (2.1), related to $u$ by (2.8), satisfies a fourth order differential equation which is homogeneous of degree two. It is written in Hirota bilinear form as

$$
D_{z}^{4} \tau \cdot \tau-\left(12 \lambda z+g_{2}\right) \tau^{2}=0
$$


where the Hirota derivative $D_{z}$ is defined by $D_{z}^{n} f \cdot g(z)=\left.\left(\frac{d}{d z}-\frac{d}{d z^{\prime}}\right)^{n} f(z) g\left(z^{\prime}\right)\right|_{z^{\prime}=z}$.

Painlevé analysis can be applied directly to the equation (4.1), expanding around a simple zero at $z=0$, corresponding to a double pole in $u$ there. Seeking resonances by taking $\tau \sim z+\varepsilon z^{r}$ yields $r=-1,0,1,6$ : the value -1 is the movable position of the singularity, as usual, and the values $r=0,1$ correspond to the two free parameters $a, b$ for the gauge transformations 2.9], which leave the equation (4.1) invariant; this leaves only $r=6$, which is equivalent to the freedom to choose $c_{6}$ (or the parameter $g_{3}$ ) in the Laurent series (2.2).

We would like to determine the Taylor series of the tau-function around $z=0$, with $\tau(0)=0$, since (due to the fact that $\tau$ is an entire function), this provides a global representation of the solution of (2.1), via the formula (2.8). If we fix the gauge, we can always choose the coefficient of $z$ to be 1 , and set the coefficient of $z^{2}$ to be 0 , which results in a series of the form

$$
\tau(z)=z+\sum_{n=2}^{\infty} C_{n} z^{n+1} .
$$

This is the analogue of the power series for the Weierstrass sigma function $\sigma(z)$, which satisfies the bilinear equation (4.1) with $\lambda=0$. Using the same method as in [3], with

$$
D_{z}^{4} z^{j} \cdot z^{k}=b_{j, k} z^{j+k-4}, \quad \text { where } \quad b_{j, k}=4 ! \sum_{\ell=0}^{4}(-1)^{\ell}\left(\begin{array}{c}
j \\
\ell
\end{array}\right)\left(\begin{array}{c}
k \\
4-\ell
\end{array}\right),
$$

it is straightforward to establish the following.

Theorem 4.1. The coefficients in the expansion (4.2) of the tau-function belong to $\mathbb{Q}\left[g_{2}, \lambda, g_{3}\right]$, being uniquely determined by the recursion

$$
n\left(n^{2}-1\right)(n-6) C_{n}=-\frac{1}{2} \sum_{j=1}^{n-1} b_{j+1, n-j+1} C_{j} C_{n-j}+\frac{1}{2} g_{2} \sum_{j=0}^{n-4} C_{j} C_{n-4-j}+6 \lambda \sum_{j=0}^{n-5} C_{j} C_{n-5-j}
$$

subject to fixing $C_{0}=1, C_{1}=0, C_{6}=-g_{3} / 840$. Each $C_{n}$ is a weighted homogeneous polynomial of total degree $n$ in the arguments $g_{2}, \lambda, g_{3}$ with weights 4,5,6 respectively.

Remark 4.2. The choice of gauge is specific to the zero at $z=0$. If we let $\tau\left(z ; g_{2}, \lambda, g_{3}\right)$ denote the function given by (4.2), and then expand around another zero at $z=\Omega \neq 0$, we obtain the formula

$$
\tau\left(z ; g_{2}, \lambda, g_{3}\right)=A e^{B(z-\Omega)} \tau\left(z-\Omega ; g_{2}+12 \lambda \Omega, \lambda, \hat{g}_{3}\right),
$$

where in principle $\hat{g}_{3}, A=\tau^{\prime}(\Omega)$ and $B=\frac{1}{2} \tau^{\prime \prime}(\Omega) / \tau^{\prime}(\Omega)$ all depend on $g_{2}, \lambda, g_{3}$ and $\Omega$. The quasiperiodicity of $\sigma(z)$ under shifting by a period is a special case of this.

For the case of the sigma function in [3] it is noted that the value of the coefficient $C_{6}$ must be given appropriately in terms of $g_{3}$ in order to be consistent with the Laurent series for $\wp$ satisfying (2.11). The same holds for $\lambda \neq 0$, upon requiring consistency of (4.2) with (2.2), as is seen by noting that the expression (2.6) for the Hamiltonian gives a first integral for the bilinear equation (4.1), taking $v=u^{\prime}$ with $u$ given by (2.8) and $h$ given by (2.10). Then the formula (2.6) can be rewritten as a third order equation for $\tau$ that is homogeneous of degree four, namely

$$
\begin{aligned}
& \tau^{2}\left(\tau^{\prime \prime \prime}\right)^{2}-6 \tau \tau^{\prime} \tau^{\prime \prime} \tau^{\prime \prime \prime}+4\left(\tau^{\prime}\right)^{3} \tau^{\prime \prime \prime}+4 \tau\left(\tau^{\prime \prime}\right)^{3}-3\left(\tau^{\prime} \tau^{\prime \prime}\right)^{2} \\
& -g_{2} \tau^{2}\left(\tau \tau^{\prime \prime}-\left(\tau^{\prime}\right)^{2}\right)-12 \lambda\left(z\left(\tau^{3} \tau^{\prime \prime}-\left(\tau \tau^{\prime}\right)^{2}\right)-\tau^{3} \tau^{\prime}\right)+g_{3} \tau^{4}=0 .
\end{aligned}
$$

This equation immediately yields another recurrence for the coefficients of $\tau$. 


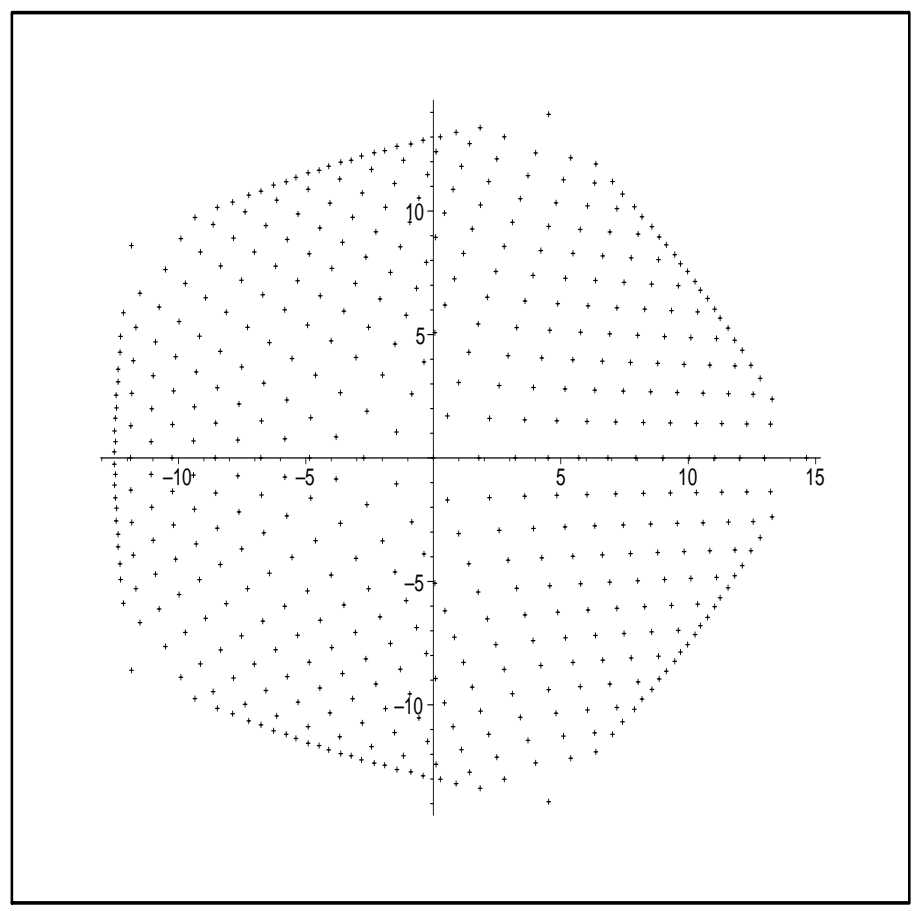

Figure 1: Approximation to the poles of the solution of 2.1) with $g_{2}=g_{3}=0$.

Theorem 4.3. Subject to fixing $C_{0}=1, C_{1}=0$, the coefficients in the expansion (4.2) are uniquely determined by the recursion

$$
n\left(n^{2}-1\right) C_{n}=-\sum_{\substack{j+k+\ell+m=n \\ 1 \leq j, k, \ell, m \leq n-1}} \tilde{b}_{j, k, \ell, m} C_{j} C_{k} C_{\ell} C_{m}+g_{2} \sum^{(4)}+12 \lambda \sum^{(5)}-g_{3} \sum^{(6)}
$$

where

$$
\tilde{b}_{j, k, \ell, m}=j(j+1)(k+1)((j-1)(k(k-6 \ell-7)+4(\ell+1)(m+1))+k(\ell+1)(4 \ell-3 m-3)),
$$

and

$$
\begin{gathered}
\sum^{(4)}=\sum_{\substack{j+k+\ell+m=n-4 \\
0 \leq j, k, \ell, m \leq n-4}}(j+1)(j-k-1) C_{j} C_{k} C_{\ell} C_{m}, \\
\sum^{(5)}=\sum_{\substack{j+k+\ell+m=n-5 \\
0 \leq j, k, \ell, m \leq n-5}}(j+1)(j-k-2) C_{j} C_{k} C_{\ell} C_{m}, \quad \sum^{(6)}=\sum_{\substack{j+k+\ell+m=n-6 \\
0 \leq j, k, \ell, m \leq n-6}} C_{j} C_{k} C_{\ell} C_{m} .
\end{gathered}
$$

Both of the recursions for $C_{n}$ given above can be iterated very rapidly to produce polynomial approximations to the tau-function, for any choice of the values $g_{2}, \lambda, g_{3}$. As an example, to generate Figure 1 we took the values $g_{2}=0=g_{3}$ and $\lambda=1$, corresponding to the solution with pentagonal symmetry that was considered in the previous section, and calculated the first 101 non-zero terms in the expansion (4.2), which contains only terms of the form $z^{5 n+1}$, beginning with $\tau(z)=z-\frac{z^{6}}{20}-$ 
$\frac{7 z^{11}}{26400}+\frac{z^{16}}{1232000}+\frac{83 z^{21}}{117976320000}+\ldots$. In the figure we have plotted the roots of the truncated series, which is of the form $P_{501}(z)=z \hat{P}_{100}(w)$, where $\hat{P}_{100}$ is a polynomial of degree 100 in $w=z^{5}$. The roots of the truncated series approximate the zeros of $\tau$ with increasing accuracy as more terms are added. The smallest root of $\hat{P}_{100}$ is a real number $\gamma$, agreeing with the digits of the numerical value (3.14) to the same accuracy as before. In fact, it appears easier to get improved approximations to this value by truncating the Taylor series than by calculating the ratios of the coefficients $c_{n}$ in the Laurent expansion.

Before concluding this section, we present another representation for the series [4.2], which displays some interesting arithmetical features of the coefficients. Note that, since the $C_{n}$ are weighted homogeneous, the tau-function can be written in the form of a triple sum

$$
\tau(z)=\sum_{\ell, m, n \geq 0} A_{\ell, m, n}\left(\frac{1}{2} g_{2}\right)^{\ell}(6 \lambda)^{m}\left(2 g_{3}\right)^{n} \frac{z^{4 \ell+5 m+6 n+1}}{(4 \ell+5 m+6 n+1) !},
$$

for certain rational numbers $A_{\ell, m, n}$. The above formula is motivated by the result of Weierstrass [22], who showed that the sigma function can be expressed as $\sigma(z)=$ $\sum_{m, n \geq 0} a_{m, n}\left(\frac{1}{2} g_{2}\right)^{m}\left(2 g_{3}\right)^{n} \frac{z^{4 m+6 n+1}}{(4 m+6 n+1) !}$. From comparison of the two series it is clear that upon setting $\lambda=0$ we have $a_{m, n}=A_{m, 0, n}$ for $m, n \geq 0$. Weierstrass used (1.2) and (2.11), together with various modular relations, to show that the sigma function satisfies two linear partial differential equations, from which he obtained a linear recurrence relation for the coefficients $a_{m, n}$. The first of these PDEs just follows from Euler's theorem on homogeneous functions, and thus extends to the tau-function when $\lambda \neq 0$ also:

$$
\left(4 g_{2} \frac{\partial}{\partial g_{2}}+5 \lambda \frac{\partial}{\partial \lambda}+6 g_{3} \frac{\partial}{\partial g_{3}}-z \frac{\partial}{\partial z}+1\right) \tau=0 .
$$

(Note that $z$ has weight -1 , so overall $\tau$ has the same weight.) However, the other PDE that Weierstrass found for $\sigma(z)$ is of second order in $z$ (containing also first derivatives with respect to $g_{2}, g_{3}$ ); it is equivalent to the fact that the corresponding elliptic theta function satisfies the heat equation. For the tau-function of $P_{I}$ we do not expect any linear PDE of this kind. Nevertheless, the Hirota bilinear equation (4.1) provides recursive relations for the coefficients $A_{\ell, m, n}$.

Theorem 4.4. Subject to fixing $A_{0,0,0}=1, A_{0,0,1}=-3$, the coefficients in (4.4) are completely determined by the recursion

$$
\begin{aligned}
& \frac{s(s-1)(s-2)(s-7)}{s !} A_{\ell, m, n}=-\frac{1}{2} \sum_{\substack{\ell_{1}+\ell_{2}=\ell \\
m_{1}+m_{2}=m \\
n_{1}+n_{2}=n}}^{\prime} \frac{b_{s_{1}, s_{2}}}{s_{1} \cdot s_{2} !} A_{\ell_{1}, m_{1}, n_{1}} A_{\ell_{2}, m_{2}, n_{2}} \\
& +\sum_{\substack{\ell_{1}+\ell_{2}=\ell \\
m_{1}+m_{2}=m-1 \\
n_{1}+n_{2}=n}} \frac{A_{\ell_{1}, m_{1}, n_{1}} A_{\ell_{2}, m_{2}, n_{2}}}{s_{1} ! s_{2} !}+\sum_{\substack{\ell_{1}+\ell_{2}=\ell-1 \\
m_{1}+m_{2}=m \\
n_{1}+n_{2}=n}} \frac{A_{\ell_{1}, m_{1}, n_{1}} A_{\ell_{2}, m_{2}, n_{2}}}{s_{1} ! s_{2} !}
\end{aligned}
$$

where $s=4 \ell+5 m+6 n+1, s_{j}=4 \ell_{j}+5 m_{j}+6 n_{j}+1$ for $j=1,2$, and $\sum^{\prime}$ denotes that the terms with $\left(\ell_{1}, m_{1}, n_{1}\right)=(0,0,0)$ or $(\ell, m, n)$ are omitted from the sum.

Remark 4.5. Another recursion for $A_{\ell, m, n}$ can be obtained from (4.3). 
The first few coefficients are given below in the form of $3 \times 3$ matrices $\mathbf{M}^{(m)}$ whose $(j, k)$ entry is $A_{j-1, m, k-1}$ for $m=0,1,2$ :

$$
\begin{gathered}
\mathbf{M}^{(0)}=\left(\begin{array}{ccc}
1 & -3 & -54 \\
-1 & -18 & 4968 \\
-9 & 513 & 257580
\end{array}\right), \quad \mathbf{M}^{(1)}=\left(\begin{array}{ccc}
-6 & -216 & 89424 \\
-84 & 18720 & 5786640 \\
1650 & 1358640 & 1168920720
\end{array}\right), \\
\mathbf{M}^{(2)}=\left(\begin{array}{ccc}
-294 & 144144 & 47585880 \\
18774 & 15053040 & 22914336240 \\
1112436 & 3160803600 & -2734614623160
\end{array}\right) .
\end{gathered}
$$

Due to recent results of Onishi [17], it is known that the coefficients $a_{m, n}$ in the expansion of the sigma function are all integers, and analogous results have been proved for sigma functions of some higher genus curves [4]. This suggests

Conjecture 4.6. The series (4.4) has $A_{\ell, m, n} \in \mathbb{Z} \quad \forall \ell, m, n \geq 0$.

We have verified this conjecture for the first few hundred coefficients.

\section{Conclusions}

The properties of the exact series expansions for the solution of $P_{I}$ and its tau-function are tantalizingly close to those of the analogous Weierstrass functions. As well as their potential uses for numerical calculations, it would be interesting to use the recursions in Theorems 4.1 and 4.3 to prove directly that $\tau$ is holomorphic (hence providing yet another proof of the fact that all solutions of $P_{I}$ are meromorphic). Finally, we should mention that all of the Painlevé equations give deformations of elliptic functions, and they all have associated tau-functions and bilinear equations [6], so the same methods could be applied to $P_{I I-V I}$. We also note that exact series formulae for $P_{V I}$ tau-functions have been obtained recently from a completely different perspective, in terms of correlation functions in 2D conformal field theory [14].

Acknowledgments. ANWH is grateful to Martin Kruskal, who suggested several years ago that it might help to consider the Taylor series for the tau-function. He also thanks Sasha Veselov for discussions at the MISGAM workshop in Berlin in 2005, concerning a similar suggestion due to Alexei Shabat. FZ wishes to acknowledge the financial support of the Istituto Nazionale di Alta Matematica, in the form of an INdAM-COFUND Marie Curie fellowship. The authors are grateful to the anonymous referee who helped to improve the manuscript.

\section{Bibliography}

[1] L. Carlitz, The coefficients of the lemniscate function, Math. Comp. 16 (1962) 475-478.

[2] J.J. Duistermaat and N. Joshi, Okamoto's space for the first Painlevé equation in Boutroux's coordinates, Archive for Rational Mechanics and Analysis 202 (2011) 707-785.

[3] J.C. Eilbeck and V.Z. Enolskii, Bilinear operators and the power series for the Weierstrass $\sigma$ function, J. Phys. A: Math. Gen. 33 (2000) 791-794.

[4] J.C. Eilbeck, Some recent results in the theory of generalized Weierstrass functions, talk at Algebrogeometric Methods in Fundamental Physics, Physikzentrum Bad Honnef, September 2012.

[5] A.S. Fokas, U. Mugan and X. Zhou, On the solvability of Painlevé I, III and V, Inverse Problems 8 (1992) 751-785. 
[6] J. Hietarinta and M. Kruskal, Hirota forms for the six Painlevé equations from singularity analysis, Painlevé Transcendents, Their Asymptotics and Physical Applications', eds. P. Winternitz and D. Levi (Plenum, 1992) 175-185.

[7] A. Hinkkanen and I. Laine, Solutions of the first and second Painlevé equations are meromorphic, $J$. d'Analyse Math. 79 (1999) 345-377.

[8] A.N.W. Hone, Painlevé Tests, Singularity Structure and Integrability, Integrability, ed. A.V. Mikhailov, Springer Lecture Notes in Physics 767 (Springer, 2009) 245-277.

[9] A. Hurwitz, Entwickelungskoeffizienten der lemniscatischen Funktionen, Math. Ann. 51 (1899) 196226.

[10] E.L. Ince, Ordinary differential equations (Dover, 1956).

[11] N. Joshi and M.D. Kruskal, A direct proof that the six Painlevé equations have no movable singularities except poles, Stud. Appl. Math. 93 (1994) 187-207.

[12] A. V. Kitaev, Symmetric solutions for the first and the second Painlevé equations, Zap. Nauchn. Sem. LOMI 187 (1991) 129-138 (Russian). English translation in J. Math. Sci. 73 (1995) 494-499.

[13] F. Lemmermeyer, Reciprocity Laws (Springer-Verlag, 2000).

[14] O. Gamayun, N. Iorgov and O. Lisovyy, Conformal field theory of Painlevé VI, J. High Energy Phys. Vol. 2012, No. 10 (2012) 38.

[15] A. Malmquist, Sur les équations différentielles du second ordre dont l'intégrale générale a ses points critiques fixes, Ark. Mat. Astr. Fys. 17 (1922-23) 1-89.

[16] K. Okamoto, On the tau-function of the Painlevé equations, Physica 2D (1981) 525-535.

[17] Y. Onishi, Universal elliptic functions. arXiv: 1003.2927

[18] J. Silverman, Advanced Topics in the Arithmetic of Elliptic Curves (Springer, 1994).

[19] N. Steinmetz, On Painlevé's equations I, II and IV, J. d'Analyse Math. 82 (2000), 363-377.

[20] N. Steinmetz, Global properties of the Painlevé transcendents: New results and open questions, Ann. Acad. Sci. Fenn. Math. 30 (2005) 71-98.

[21] E.C. Titchmarsh, The theory of functions (Oxford University Press, 2nd edition, 1939).

[22] K. Weierstrass, Zur Theorie der elliptischen Funktionen, Mathematische werke von Karl Weierstrass herausgegeben unter Mitwirkung einer von der Königlich preussischen Akademie der Wissenschaften eingesetzten Comission 2 (1894) 245-255. Originally published in Sitzungsberichte der Akademie der Wissenschaften zu Berlin (1882) 443-451.

[23] E.T. Whittaker and G.N. Watson, A course of modern analysis (Cambridge University Press, 1927). 